\title{
Cutaneous Larva Migrans in Travelers: Synopsis of Histories, Symptoms, and Treatment of 98 Patients
}

\author{
T. Jelinek, H. Maiwald, H. D. Nothdurft, and T. Löscher
}

\author{
From the Department of Infectious Diseases and Tropical Medicine,
} University Hospital, University of Munich. Munich. Germany

\begin{abstract}
The symptoms, medical history, and treatment of 98 patients with cutaneous larva migrans (creeping eruption) who attended a travel-related-disease clinic during a period of 4 years are reviewed. This condition is caused by skin-penetrating larvae of nematodes, mainly of the hookworm Ancylostoma braziliense and other nematodes of the family Ancylostomidae. Despite the ubiquitous distribution of these nematodes, in the investigated group only travelers to tropical and subtropical countries were affected; $28.9 \%$ of the patients had symptoms for $>1$ month, and for $24.5 \%$ the probable incubation period was $>2$ weeks. The efflorescences typically were on the lower extremities (73.4\% of all locations). The buttocks and anogenital region were affected in $12.6 \%$ of all locations, and the trunk and upper extremities each were affected in $7.1 \%$. Only a minority of patients presented with eosinophilia or an elevated serum level of IgE. No other laboratory data appeared to be related to the disease. Therapy with topical thiabendazole was successful for $\mathbf{9 8 \%}$ of the patients. Systemic antihelmintic therapy was necessary in two cases because of disseminated, extensive infection.
\end{abstract}

The syndrome of cutaneous larva migrans develops when certain skin-penetrating larvae migrate into the dermis and cause distinct symptoms. These are mainly characterized by tunnel-like migrating erythemas with severe pruritus. The name creeping eruption is derived from these signs. Usually the causative agents are larvae of animal parasites, which infect humans (as an abnormal host) without reaching sexual maturity. Following penetration, the larvae stay in the stratum germinativum. The active production and secretion of hyaluronidase seem to render the movements of the larvae possible [1]. Usually a typical dermatitis with vesiculae, papules, and desquamations develops within a few days [figure 1]. The typical erythema occurs $3-4 \mathrm{~cm}$ away from the penetration site. The larva itself is usually $1-2 \mathrm{~cm}$ ahead of the creeping eruption. Within weeks or sometimes months of invasion, the larva dies and is resorbed [2]. The infection is usually restricted to the skin. In rare cases invasion of the lungs, followed by the development of a pulmonary eosinophilic infiltrate and the presence of larvae in lavage fluid, has been reported [3-5].

Most common is infection by the larvae of Ancylostoma braziliense, a ubiquitous hookworm found in dogs, cats, and other mammals. Ancylostoma caninum and Uncinaria stenocephala, other hookworms parasitic in dogs and cats, can also cause cutaneous larva migrans $[4,6,7]$. From animal feces the eggs are passed into the soil, where the larvae hatch. They

Received 16 December 1993; revised 31 May 1994.

Reprints or correspondence: Dr. Tomas Jelinek. University of Munich. Department of Infectious Diseases and Tropical Medicine, Leopoldstr. 5 , 80802 München, Germany.

Clinical Infectious Diseases 1994;19:1062-6

(C) 1994 by The University of Chicago. All rights reserved. |058-4838/94/1906-00। I \$02.00 initially feed on soil bacteria, molt twice before the infective third stage, and penetrate through the skin of the new host $[4,6,7]$. All unprotected parts of the body in contact with the soil are at risk. On rare occasions the disease might be caused by Gnathostoma spinigerum, a nematode endemic in Asia; Dirofilaria species; and Strongyloides procyonis, a parasite of raccoons [6-8] that is thought to be responsible for the seasonal "duckhunters" itch" among North American hunters [7]. Creeping eruption is usually a pathognomonic sign of cutaneous larva migrans. Important differential diagnoses are larva currens, visceral larva migrans, myiasis, scabies, and the erythema migrans of Lyme disease $[8,9]$.

The objective of this investigation was to gain information about the regions where cutaneous larva migrans is most frequently acquired by German travelers; about the character, onset, and duration of symptoms; and about treatment before presentation. Furthermore, data concerning the immunologic reaction of the patients were included in the analysis whenever available.

\section{Methods}

Of 13,300 patients visiting the travel-related-disease clinic of the University of Munich between January 1990 and October $1993,11 \%$ presented with skin symptoms. Cutaneous larva migrans was diagnosed clinically in 98 patients (6.7\% of the patients with skin symptoms). The charts of all $98 \mathrm{pa}-$ tients were reviewed in regard to medical history, symptoms, and therapy. All patients were German nationals returning from vacations abroad; $54(55.1 \%)$ were male, and 44 (44.9\%) were female. The majority ( $74.5 \%$ ) were aged $21-40$ years (mean, 32 years). Laboratory methods used were parasitological stool investigations (Merthiolate [thimerosal]iodine-formalin concentration; MIFC) [10], determinations 


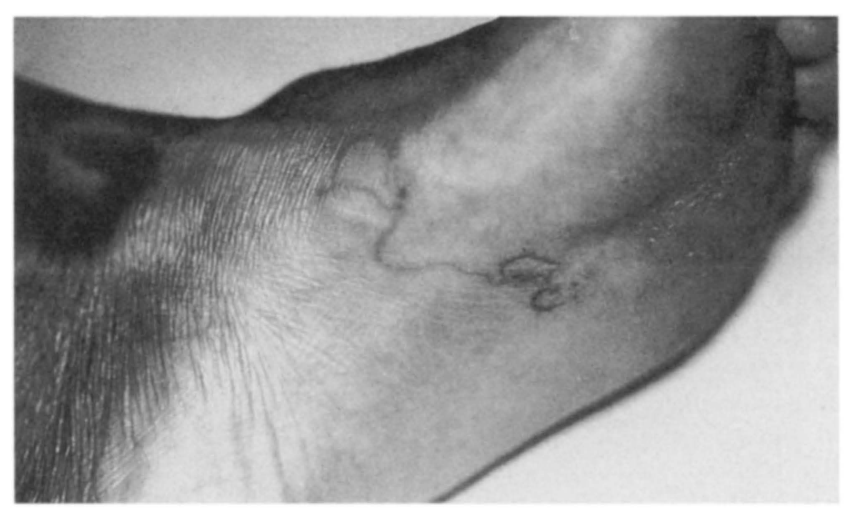

Figure 1. This photograph of the foot of a patient with cutaneous larva migrans illustrates the typical skin signs associated with creeping eruption.

of levels of eosinophiles and total serum IgE, and ELISAs for antibodies to Toxocara canis (excretory/secretory antigens) [11], Dirofilaria immitis, and Onchocerca volvulus (adult worm antigens) [12].

\section{Results}

Duration of the patients' travel varied from 1 to 72 weeks (mean, 6 weeks). Of their destinations, Southeast Asia clearly headed the list (31 patients [31.6\%]), followed by the Caribbean (19 [19.4\%]), South America (13 [13.4\%]), East Africa and the Indian subcontinent (10 [10.2\%] each), Central America (8 [8.2\%]), and West Africa (5 [5.1\%]). There was a markedly low incidence of travel to South and North Africa (1 [1\%] each) (figure 2).

For 53 patients (54.1\%), no exact data were available regarding the duration of their symptoms. Of the remaining 45 patients, $28.9 \%$ reported having symptoms for $>1$ month, and one patient's symptoms lasted 9 months (range, 1-36 weeks; mean, 5.6 weeks). Of the 98 patients, $75.5 \%$ presented with the typical symptoms of creeping eruption within 4 weeks of returning from their journey, and 24.5\% were seen $>4$ weeks after returning (range, 1-36 weeks; mean, 5 weeks); in two of the latter cases, presentation occurred 9 months after return. From the available data, the time of onset of symptoms could be determined for 45 patients. Two patients' symptoms began 7 months after their return. The time of onset ranged from 16 weeks before return from the journey to 28 weeks after return (mean, 1.5 days after return).

In all cases the diagnosis was established instantly from the case history and typical symptoms. Twenty-two patients presented with cutaneous larva migrans in more than one area of the body. The lower extremities were affected in most cases (73.4\% of all locations): eruptions on the feet represented $61.7 \%$ of all locations; on the lower legs, $3.1 \%$; and on the upper legs, $8.6 \%$. Eruptions on the buttocks and/or anogenital region represented $12.6 \%$ of all locations; these eruptions were usually in areas typically covered by bathing suits (figure 3). Creeping eruptions on the trunk represented 7.1\% of all locations, and eruptions of the upper extremeties represented another $7.1 \%$ (most of the latter eruptions occurred in small children) (figure 4). Symptoms did not involve the head, but in rare cases such symptoms have been described in the literature [13].

Laboratory findings played no role in the establishment of the diagnosis. Only $20 \%$ of the 40 patients tested had eosinophilia, defined as an eosinophil proportion of $\geqslant 7 \%$ of the leukocyte count (eosinophil proportion range, 0\%-37\%; mean, 5\%). The total serum level of IgE, which was measured in 13 patients, ranged from 13 to $4,000 \mathrm{IU} / \mathrm{mL}$ (mean, 539 $\mathrm{IU} / \mathrm{mL}$ ); six patients had IgE levels $>200 \mathrm{IU} / \mathrm{mL}$, but the majority (seven patients) had levels below this mark. Elevated titers of antibodies to the $T$. canis ES-antigen were demonstrated in four of 10 patients tested; factors (multiples
Figure 2. This map indicates areas of the world to which 98 patients with cutaneous larva migrans had traveled prior to the onset of their symptoms: Southeast Asia (31.6\%), the Caribbean or Central America (27.6\%), South America (13.4\%), East Africa and the Indian subcontinent (10.2\% each), West Africa $(5.1 \%)$, and South Africa and North Africa (1\% each).

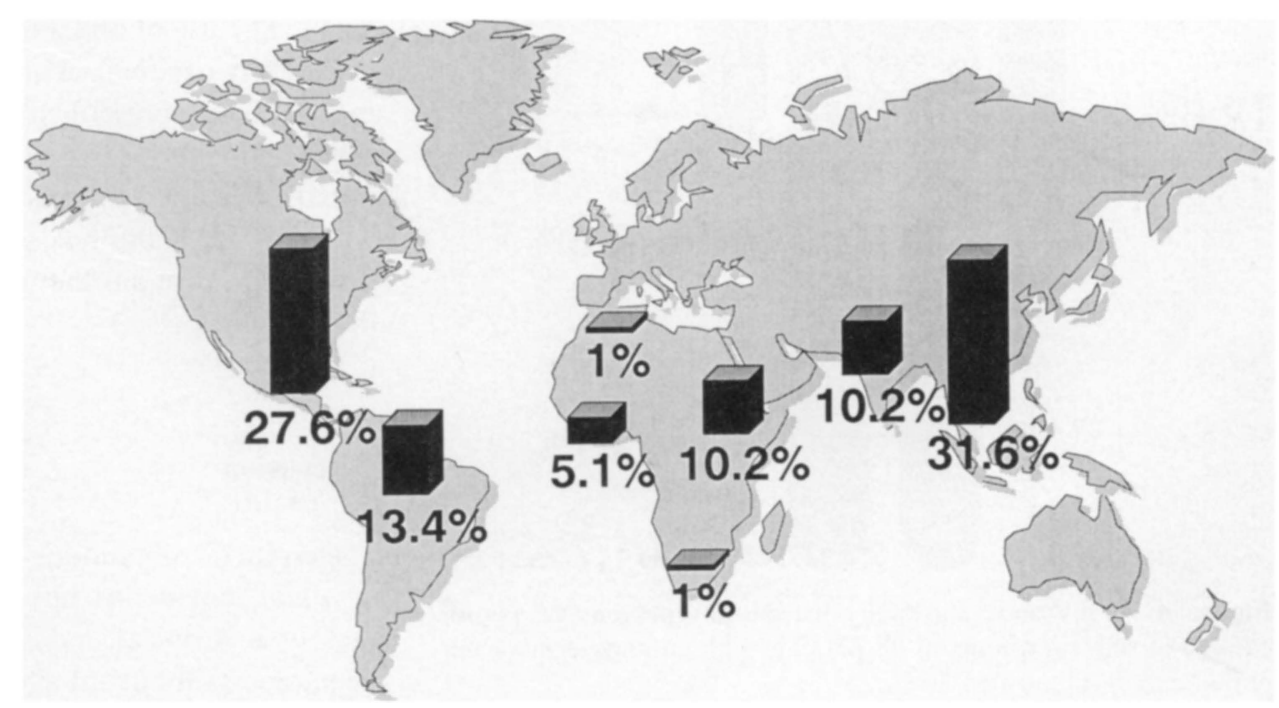




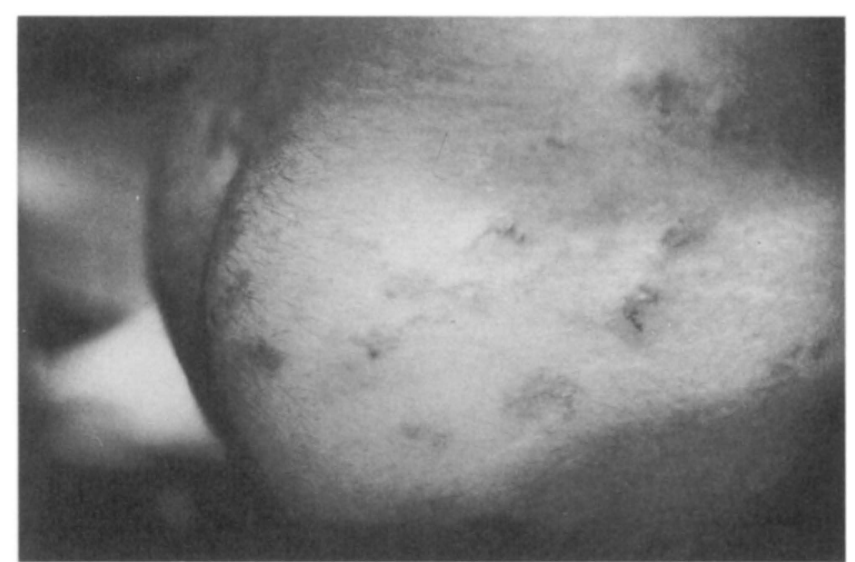

Figure 3. Severe creeping eruption was noted on the buttocks of a patient with cutaneous larva migrans who had recently returned from Southeast Asia.

of normal activity) ranged from negative values to 41 (mean factor, 12). Significant titers of filarial antibodies were detected in only one of 18 patients tested. Stool analysis was performed for 28 patients but revealed helminthic infection in none.

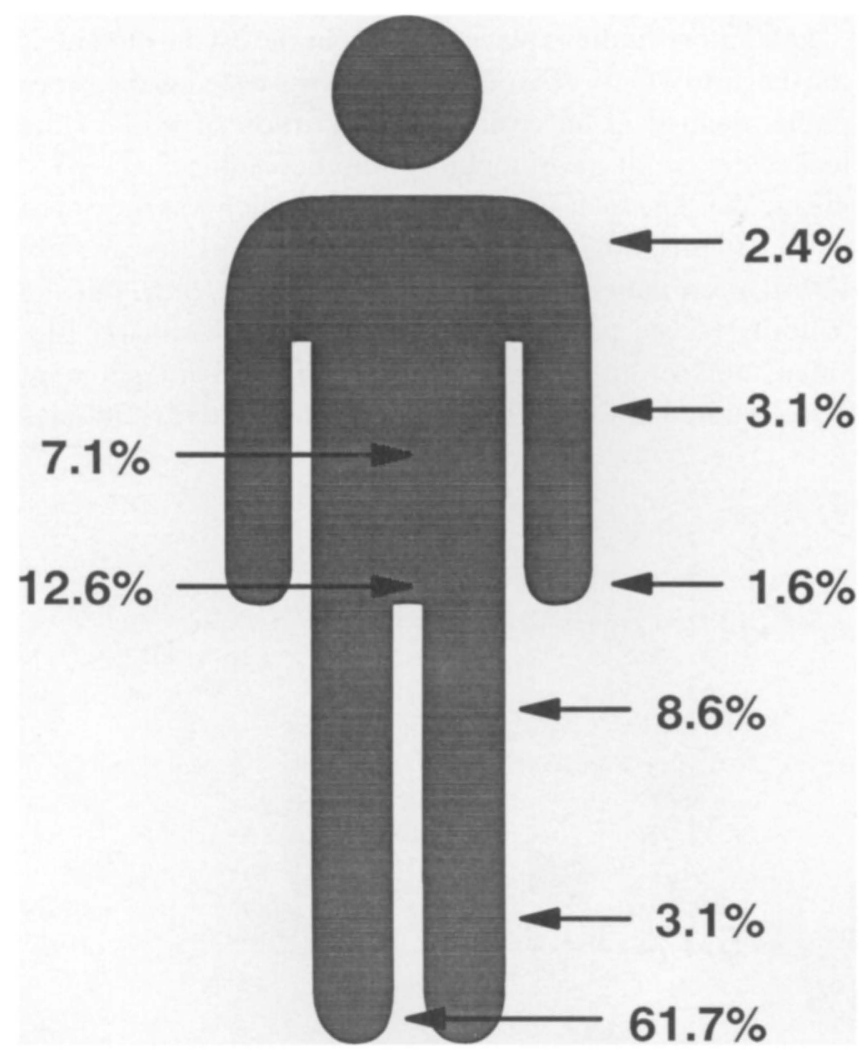

Figure 4. This model shows the distribution (percentages per location) of skin symptoms in 98 patients with cutaneous larva migrans.
Table 1. Treatment received by 22 patients with cutaneous larva migrans before presentation at the hospital clinic. Some patients received more than one type of treatment.

\begin{tabular}{lc}
\hline Agent or method of treatment & No. of patients pretreated \\
\hline Thiabendazole ointment & 4 \\
Soap & 1 \\
French brandy (external) & 1 \\
Surgery & 3 \\
Fluid nitrogen & 6 \\
Antihistamines (oral) & 3 \\
Antibiotics (oral) & 4 \\
Mebendazole (oral) & 2 \\
Antimycotic ointments & 2 \\
Unspecific ointments & 7 \\
Antiscabiotic ointments & 2 \\
Antibiotic ointments plus & \\
steroid ointments & 2 \\
\hline
\end{tabular}

At presentation, $22(22.4 \%)$ of the patients had already received some kind of treatment. Frequently, several methods had been alternated or used simultaneously in the treatment of a patient (table 1). Altogether, 12 different methods of treatment were used. Most commonly, nonspecific ointments were prescribed. For six patients, cryotherapy (with repeated use of fluid nitrogen) was used in an attempt to kill the larvae. This method was unsuccessful for all patients and resulted in severe blistering or ulceration of the skin of two patients. Just as unsuccessful were surgical intervention (3 patients), locally applied antiscabiotic ointments (2 patients), and oral mebendazole ( 2 patients). Four patients had received thiabendazole ointment for local application. In these cases the symptoms, notably the itching, had usually already ceased. At the travel-related-disease clinic, all 98 patients were treated with thiabendazole ointment applied locally. In all cases we used a special preparation of $15 \%$ thiabendazole and 3\% salicylic acid in unguentum alcoholum lanae. The use of oral antihelmintic agents as well was indicated for two patients with disseminated, extensive infection; in one case we prescribed a single dose of albendazole, in the other a single dose of thiabendazole. No side effects were noted. The therapy was successful for 96 patients (98\%) within 10 days. In one case treatment had to be extended to 2 weeks, and in another case to 4 weeks, before the lesions disappeared.

\section{Discussion}

Despite the ubiquitous distribution of $A$. braziliense and $A$. caninum, cutaneous larva migrans seems to be restricted mainly to tropical and subtropical countries. There have been reports about autochthonous infections in northern cli- 
mates, but these cases seem to be rare $[2,4,6,7]$, as was indicated by the results of our investigation. None of the investigated patients had acquired infection at any of the destinations more popular among European tourists, in the northern and eastern Mediterranean, despite the fact that $A$. braziliense and other nematodes of the Ancylostomidae family are quite endemic in these areas [8]. However, only $2.2 \%$ of our total patient population had traveled to Mediterranean countries before referral. This might present a considerable bias for speculations about the frequency of larva migrans infection in these areas.

In the investigated series of patients, the course of cutaneous larva migrans frequently was chronic; $28.9 \%$ of the patients for whom such data were available reported that the duration of symptoms was $>1$ month, and for one patient it was 9 months. These patients frequently had received various nonspecific treatments, often with fluid nitrogen, which had obviously not shortened the course of disease.

The probable incubation period was estimated by calculating the difference between the time of return to Germany and the time of onset of symptoms. This method might have led to underestimations in some cases, because it imputes the time of infection to the last day of the journey. Even so, for $24.5 \%$ the probable incubation period was $>2$ weeks, while those reported in the literature vary from minutes to a few days $[2,6,7]$. Two patients developed symptoms 7 months after returning from their journey. The possibility that infection occurred in Germany should be considered in these cases. It cannot be excluded completely, although case reports of infections in northern climates are rare $[4,6]$. However, considering the high percentage of our patients for whom the onset of symptoms was delayed, we favor the theory that infection occurred during the journey, especially since none of the patients mentioned extensive exposure to possibly contaminated soil in Germany.

The frequency with which the buttocks, anogenital region, trunk, and upper extremities were affected is notable. It seems likely that the patients whose buttocks and genitalia were affected were nude in the sand for some time. It is notable that the upper extremities were affected more often in children than in adults. The most likely explanation for this phenomenon is that these areas in small children frequently are exposed to soil for extended periods.

The differentiation between scabies and cutaneous larva migrans is not always easy, especially if the latter presents with atypical efflorescences on the upper extremities. Important diagnostic criteria for scabies are the following: a history of exposure to surroundings possibly infested by the mite Sarcoptes scabiei; crustaceous lesions, papules, generalized itching, and rash; microscopic demonstration of S. scabiei, its eggs, or its feces in skin scrapings; involvement of the hands; and the appearance of multiple lesions. In cases of cutaneous larva migrans, the appearance of single migrating tracts and vesicular lesions, involvement of the legs rather than the hands, and rapid cessation of symptoms after the use of thiabendazole ointment are important diagnostic clues.

Our data did not confirm that eosinophilia and a rise in the total serum titer of $\mathrm{IgE}$ are typical signs of cutaneous larva migrans as they are in systemic helminthic infections [2, 4]. Perhaps the presentation of antigen to the immune system is limited in infections that usually are confined to the skin. The overwhelming majority of our laboratory results were within the normal range. Therefore, the diagnosis of cutaneous larva migrans seems to rely solely on clinical findings. Laboratory findings might support the diagnosis but usually cannot prove it.

Surgical therapy was attempted for three patients, in all cases without success. Since the larva is situated $1-2 \mathrm{~cm}$ ahead of the visible track, it cannot be localized reliably and therefore can only rarely be removed by a knife [2, 4]. Cryotherapy with the application of fluid nitrogen led to blisters and chronic ulcerations in two of the six patients treated by this method, and subsequently all six patients' creeping eruptions were still spreading. This finding underlines the fact that the larvae are capable of surviving temperatures as low as $-21{ }^{\circ} \mathrm{C}$ for $>5$ minutes [8]. Therefore, we do not recommend cryotherapy for treatment of cutaneous larva migrans, although the group treated in this way is too small to allow statistical analysis.

For $98 \%$ of the investigated patients, cure was achieved within 10 days by the topical application of thiabendazole ointment only. For two patients with extensive, disseminated infections, treatment with single oral doses of thiabendazole and albendazole was preferred [13-17]. The frequently severe itching ceased rapidly for all patients following the application of antihelmintic agents. These results demonstrate the excellent efficacy of topically applied thiabendazole in the treatment of cutaneous larva migrans.

\section{References}

1. Hotez PJ, Narasimhan S, Haggerty J, et al. Hyaluronidase from infective Ancylostoma hookworm larvae and its possible function as a virulence factor in tissue invasion and in cutaneous larva migrans. Infect Immun 1992; 60: 1018-23.

2. Katz R, Ziegler J, Blank $H$. The natural course of creeping eruption and treatment with thiabendazole. Arch Dermatol 1965;91:420-4.

3. Ash LR. Larva migrans then. Am J Trop Med Hyg 1989;41(3)(suppl): 18-20.

4. Edelglass JW, Douglass MC, Stiefler R, Tessler M. Cutaneous larva migrans in northern climates: a souvenir of your dream vacation. $\mathbf{J}$ Am Acad Dermatol 1982:7:353-8.

5. Guill MA, Odom RB. Larva migrans complicated by Loeffler's syndrome. Arch Dermatol 1978; 114:1525-6.

6. Herbener D, Borak J. Cutaneous larva migrans in northern climates. Am J Emerg Med 1988; 6:462-4.

7. Davies HD, Sakuls P, Keystone PS. Creeping eruption. A review of 
clinical presentation and management of 60 cases presenting to a tropical disease unit. Arch Dermatol 1993; 129:588-91.

8. Elliot DL. Tolle SW, Goldberg L, Miller JB. Pet-associated illness. N Engl J Med 1985;313:985-95.

9. van Knapen F, Buijs J, Kortbeek LM, Ljungström I. Larva migrans syndrome: toxocara, ascaris or both? [letter]. Lancet 1992;340: 550-1.

10. Basic laboratory methods in medical parasitology. Geneva: World Health Organization, 1991.

11. Sugane K. Oshima T. Purification and characterization of excretory and secretory antigen of Toxocara canis larvae. Immunology 1983; 50:113-20.

12. Bartlett A. Bidwell DE, Voller A. Preliminary studies on the application of enzyme immunoassay in the detection of antibodies in onchocerciasis. Tropenmed Parasitol 1975;26:370-4.

13. André J, Bernard M, Ledoux M, Achten G. Larva migrans of the oral mucosa. Dermatologica 1988; 176:296-8.

14. Orihuela AR, Torres JR. Single dose of albendazole in the treatment of cutaneous larva migrans [letter]. Arch Dermatol 1990; 126:398-9.

15. Whiting DA. The successful treatment of creeping eruption with topical thiabendazole. S Afr Med J 1976;50:253-5.

16. Jones SK, Reynolds NJ, Oliwiecki S, Harman RRM. Oral albendazole for the treatment of cutaneous larva migrans. $\mathrm{Br} \mathrm{J}$ Dermatol 1990; 122:99-101.

17. Hepburn D, Nicholls DSH. Treatment of cutaneous larva migrans [letter]. N Z Med J 1990; 103:518. 